\title{
A CHARACTERIZATION OF UNIONS OF TWO STAR-SHAPED SETS
}

\author{
C. F. KOCH AND J. M. MARR
}

We shall use the notation $S(x)$ to denote the star of $x$ in $M$, that is, the set of all points $y \in M$ such that the segment $x y$ is contained in $M$. The star (in $M$ ) of a set $A$ is defined by $S(A)=\mathrm{U}_{x \in A \cap M} S(x)$. We use the terminology "the point $x$ sees the point $y$ " to mean the closed segment $x y$ is contained in $M$.

Let $M$ be a closed set in $E_{r}$. Suppose there exists a line segment $S_{1}$ such that each triple of points $x, y, z$ in $M$ determines at least one point $p \in S_{1}$ such that at least two of the points $x, y, z$ see the point $p$ via $M$. Valentine $[1$, Problem 6.6, p. 178] has conjectured that this property characterizes $M$ as the union of at most two star-shaped sets. That this condition is necessary follows immediately by choosing $S_{1}$ to be any line segment which intersects the kernels of the two starshaped sets. A further property which is enjoyed by every union of two star-shaped sets is the following.

Condition A. If $S_{1}=\bigcup_{i=1}^{m} I_{i}$ where the $I_{i}$ are closed intervals with at most end points in common, then of the intervals $I_{i}$ there is at least one pair (say $I_{r}$ and $I_{s}$ ) such that at least two of every triple of points of $M$, see a common point of $I_{r} \cup I_{s}$ via $M$.

If it is true that of each triple of points of $M$ at least two of them see a common point of a single interval (say $I_{r}$ ), then the pair $I_{r}$ and $I_{s}$ where $I_{s}=I_{r}$ satisfies the conclusion of condition $A$. The reader will note that if $m=2$, then Condition A implies Valentine's property.

Next we note that a set $M$ satisfying either Valentine's property or Condition A consists of at most two components, for if $M$ had as many as three components the selection of a point from each of the components would violate Valentine's condition. In the two component case Valentine's condition can be stated as follows. If $x$ and $y$ are in the same component of $M$, then there exists a point $p \in S_{1}$ such that the segments $x p$ and $y p$ are contained in $M$. But then an application of a generalization of Krasnosel'skir's theorem [1, Theorem 6.18, p. 85] tells us that each component is star-shaped. Before proceeding with the case in which $M$ is connected we prove the following lemma.

Lemma. Suppose $M$ is connected and $A$ is a compact subset of $S_{1}$ such

Received by the editors January 25, 1966 and, in revised form, April 15, 1966. 
that for each three distinct points $x, y, z$ of $M$ at least two of these points see a common point $p$ of $A$. Then $M=S(A)$.

Proof. Let $q \in M$. Since $M$ is connected, there exist sequences $\left\{x_{n}\right\}$ and $\left\{y_{n}\right\}$ which converge to $q$ and such that for each $n$, the points $x_{n}, y_{n}$ and $q$ are distinct. The hypothesis implies that, for each $n$, at least one of the points $x_{n}$ or $y_{n}$ sees a point $p_{n} \in A$. Since $A$ is compact, a subsequence $\left\{p_{n}^{\prime}\right\}$ of $\left\{p_{n}\right\}$ can be found which converges to $p_{0} \in A$ together with a corresponding subsequence (say $\left\{x_{n}^{\prime}\right\}$ ) of one of the sequences $\left\{x_{n}\right\}$ or $\left\{y_{n}\right\}$ such that $x_{n}^{\prime} p_{n}^{\prime} \subset M$. Then every point of $q p_{0}$ is a limit point of points on the segments $x_{n}^{\prime} p_{n}^{\prime}$, and since $M$ is closed, $q p_{0} \subset M$. Thus $M=S(A)$.

THEOREM. Let $M$ be a closed subset of $E_{r}$ which satisfies Condition A with respect to a line segment $S_{1}$. Then $M$ is the union of at most two star-shaped sets.

Proof. Assume that $M$ is connected since the case where $M$ consists of two components has already been discussed. For each positive in teger $k$, divide $S_{1}$ in to $2^{k}$ closed subintervals $I_{j}\left(j=1,2, \cdots, 2^{k}\right)$ of equal length and with at most end points in common. Then Condition A guarantees the existence of a pair of these intervals $I_{r}$ and $I_{s}$ such that at least two of every triple of points of $M$ see a common point of $I_{r} \cup I_{s}$ via $M$. If for any $k$, it is possible to choose a pair $I_{r}$ and $I_{s}$ with $I_{s}=I_{r}$ and still preserve this property, we do so. By the lemma, $M=S\left(I_{r}\right) \cup S\left(I_{s}\right)$. Then for each $k$, define $I_{r}=I_{k}$ and $I_{s}=J_{k}$. It follows that for each $k, M=S\left(I_{k}\right) \cup S\left(J_{k}\right)$.

For each $k$, select points $x_{k} \in I_{k}$ and $y_{k} \in J_{k}$. Since $S_{1}$ is compact, we select subsequences $\left\{x_{n}\right\}$ of $\left\{x_{k}\right\}$ and $\left\{y_{n}\right\}$ of $\left\{y_{k}\right\}$ which converge to $x_{0} \in S_{1}$ and $y_{0} \in S_{1}$ respectively.

We now show $M=S\left(x_{0}\right) \cup S\left(y_{0}\right)$. Let $p$ be an arbitrary point of $M$. By the lemma and the definitions of $I_{n}$ and $J_{n}, p$ sees a point $z_{n} \in I_{n} \cup J_{n}$ for each $n$. Without loss of generality we may assume that $p$ sees $z_{n} \in I_{n}$ for infinitely many $n$. Let $\epsilon$ be any positive number and define $U$ and $V$ to be the respective intersections of $S_{1}$ with $\epsilon$ and $\epsilon / 2$ spherical neighborhoods of $x_{0}$. Since $\left\{x_{n}\right\}$ converges to $x_{0}$, there exists an integer $N_{1}$ such that if $n>N_{1}, x_{n} \in V$. We select the integer $N_{2}$ such that if $n>N_{2}$, the length of $I_{n}$ is less than $\epsilon / 2$. Then for infinitely many $n>\max \left(N_{1}, N_{2}\right), I_{n} \subset U$ and $p z_{n} \subset M$. Since $p$ sees a point in every neighborhood of $x_{0}$ and $M$ is closed, the segment $p x_{0} \subset M$. Thus each point of $M$ sees at least one of the points $x_{0}$ or $y_{0}$ via a segment in $M$. If for every $n, I_{n}=J_{n}$, then $x_{0}=y_{0}$ and $M$ is starshaped. If $x_{0} \neq y_{0}$, then $M$ is the union of two star-shaped sets. Thus the proof is complete. 
The following example shows that the assumption that $M$ is closed cannot be deleted. Consider the union of two disjoint closed discs in the plane together with the segment joining their centers. From each disc delete all the points of a diameter not parallel to the line of centers excepting the end points and the center itself. The set described is $M$ and $S_{1}$ is the intersection of the line of centers with $M$. Then $M$ is not closed, satisfies Valentine's condition and Condition A, but it is not the union of two star-shaped sets.

\section{REFERENCE}

1. F. A. Valentine, Convex sets, McGraw-Hill, New York, 1964.

Kansas State University 